\title{
Modifications radioinduites du relief cutané : résultats préliminaires chez le porc
}

\author{
J.L. LEFAIX*, J. MIGNOT**
}

(Manuscrit reçu le 4 juillet 1994,

revisé le 2 septembre 1994, accepté le 11 octobre 1994)

RÉSUMÉ Le relief cutané évolue sous l'action de nombreux paramètres physiologiques et physico-chimiques comme le sexe, lâge, l'hydratation et les rayonnements. Composée de plateaux traversés de vallées, la surface cutanée peut être étudiée par une analyse $3 D$ de sa topographie et par une analyse profilométrique $2 D$. Cette étude préliminaire de la topographie de la peau irradiée met en évidence une diminution relative des paramètres décrivant l'amplitude verticale $(\sim 15 \%)$ du relief cutané $24 \mathrm{~h}$ après irradiation et quelle que soit la dose, chez 3 porcs ayant reçu une dose unique de $\beta$ du ${ }^{90} \mathrm{Sr}-90 \mathrm{Y},\left(32,64\right.$ et $96 \mathrm{~Gy}$ sous $7 \mathrm{mg} / \mathrm{cm}^{2}$; distance source - peau : $2,6 \mathrm{~cm}$ ) et un porc ayant reçu une dose unique de $\gamma$ du ${ }^{192} \mathrm{Ir}$ (160 Gy ; distance source - peau : 1,7 cm). Ce résultat préliminaire sera complété par une étude sur une série chronologique portant sur plusieurs individus afin de définir le seuil de dose d'irradiation induisant des modifications du relief cutané, avant d'être validé chez l'homme.

ABSTRACT The evolution of the cutaneous relief depends on physiological and physical parameters such as sex, ageing, hydratation and radiations. Composed of plateaus crossed by valleys, the skin surface topography can be studied by a $3 \mathrm{D}$ topographic and a 2D profilometric analysis of the distribution of the relief parameters. This preliminary study of the irradiated skin topography showed a relative decrease of the vertical amplitude parameters $(\sim 15 \%)$ of the skin relief in three pigs irradiated with single doses of ${ }^{90} \mathrm{Sr}-{ }^{90} \mathrm{Y} \beta$ rays $\left(32,64\right.$ and $96 \mathrm{~Gy}$ under $7 \mathrm{mg} / \mathrm{cm}^{2}$; skin source distance : $2.6 \mathrm{~cm})$ and one pig irradiated with a single dose of ${ }^{192} \mathrm{Ir} \gamma$ rays $(160 \mathrm{~Gy}$ skin surface dose ; skin source distance : $1.7 \mathrm{~cm}$ ), $24 \mathrm{~h}$ after irradiation whatever the dose. This preliminary result will be developed in a larger study group of pigs and a longer time period, to define the threshold dose of irradiation that induces modifications of the skin topography, before being validated in humans.

\section{Introduction}

Les applications industrielles et médicales des rayonnements ionisants se sont considérablement développées ces vingt dernières années [22]. Cependant, lors d'une irradiation accidentelle, l'évaluation dosimétrique précoce et précise

* Commissariat à l'énergie atomique, Direction des sciences du vivant, Département de pathologie et de toxicologie expérimentales, DSV-DPTE, Laboratoire de radiobiologie appliquée, CESaclay, 91191 Gif-sur-Yvette Cedex.

** Laboratoire de métrologie des interfaces, IUT, BP 1559, 25009 Besançon Cedex. 
des lésions aiguës localisées est un problème encore non résolu aujourd'hui en ce qui concerne la peau et les tissus sous-cutanés. Le clinicien est généralement placé devant une symptomatologie fugace et souvent discrète, dont l'évolution dans les semaines et les mois qui suivent l'accident peut entraîner des délabrements tissulaires très importants. Au niveau de la peau et des tissus sous-cutanés, les méthodes atraumatiques d'explorations paracliniques qui permettraient de distinguer un tissu sain d'un tissu irradié, afin d'en délimiter l'étendue en surface et en profondeur, sont nombreuses et peuvent être classées en deux types de méthodes [8], caractérisant les modifications :

- des propriétés biophysiques (densité et hydratation tissulaire, abordées par des techniques de tomographie aux rayons $\mathrm{X}$, imagerie en résonance magnétique nucléaire (IRM) et échographie ultrasonore) ;

- de l'état physio-pathologique (circulation cutanée et sous-cutanée, abordées par des techniques de mesure de l'effusivité, de conductivité thermique, de thermographie infrarouge et microonde, de débit sanguin local et de mesures transcutanées de la pression partielle d'oxygène).

Cependant, l'évaluation de la validité de ces méthodes chez l'homme est difficile en raison d'un nombre de patients accidentés fort heureusement très faible: le nombre de relations dose-effets bien établies au plan cutané reste ainsi très limité. L'expérimentation animale paraît donc la méthode de choix, particulièrement chez le porc dont la peau peu pourvue de poils, l'irrigation cutanée et sous-cutanée et la vitesse de renouvellement cellulaire de l'épiderme sont proches de ceux de l'homme et ont déjà fait l'objet de nombreux travaux en radiobiologie cutanée [10-11].

Dans un modèle expérimental d'accident de surexposition en gammagraphie industrielle, développé chez le porc au moyen d'une source d'iridium 192, nous avons pu établir des relations entre l'intensité de la réponse enregistrée en thermographie microonde et la gravité de la lésion cutanéo-musculaire $[6,13]$, la visualisation précoce de l'cedème musculaire en IRM et l'anatomopathologie extemporanée $[7,14]$, et démontrer l'intérêt d'une intervention chirurgicale précoce et a minima après des irradiations aiguës localisées supérieures à $100 \mathrm{~Gy}$ à la peau [15-16]. Par ailleurs, nous avons pu établir une relation entre la dose d'irradiation à la peau et l'extension de la fibrose radioinduite tardive [16]. Plus récemment, des modifications très précoces du signal IRM du tissu cutané ont été mises en évidence pour des doses d'irradiation de 32 à 96 Gy par Chalansonnet [2].

L'étude du microrelief cutané est une technique bien connue en dermatologie et en cosmétique, depuis l'étude de Makki et al. [18] qui, les premiers, avaient montré l'intérêt de la profilométrie et de la microtopographie de la peau humaine. Ce travail original faisait suite à des travaux pionniers de R. Marx qui tentait d'élucider les mystères des variations du stratum corneum. En fait, le relief cutané évolue sous l'action de nombreux paramètres comme l'âge, le sexe, le siège anatomique, le degré d'hydratation, l'influence de certaines pathologies et d'agressions physico-chimiques. Schématiquement, les 
méthodes employées pour l'étude quantitative du relief cutané relèvent de la profilométrie [4-5] dérivée de la profilométrie industrielle. Cependant, composé de plateaux traversés de sillons, le relief cutané ne peut être quantifié correctement en utilisant les paramètres traditionnels créés pour l'analyse des surfaces industrielles [12].

Aujourd'hui, l'analyse bidimensionnelle ou profilométrique du relief de la peau humaine est basée sur une décomposition spectrale respectant l'existence de deux familles de plis, l'une liée au derme profond, l'autre au stratum corneum et à l'épiderme. L'analyse statistique, initialement dédiée à l'étude de la répartition des vagues à la surface de la mer, permet ainsi de mesurer l'anisotropie du relief. De même, l'analyse tridimensionnelle permet d'une part de quantifier la distribution des hauteurs du relief d'un point de vue statistique et morphologique et, d'autre part, de relever la répartition et la densité des plis dans différentes directions de la surface [24]. Ces méthodes de traitement du relief cutané ont trouvé de nombreuses implications dans des études de cicatrisation d'ulcères chroniques des jambes [26] ou de vieillissement cutané [23].

L'étude du relief cutané humain $24 \mathrm{~h}$ après une exposition aux UV-B a permis à Bessis et al. [1] d'observer des modifications proportionnelles à la dose délivrée. De même, les effets à long terme consécutifs à des expositions uniques ou fractionnées ont été bien étudiées grâce au suivi de la profilométrie. Il a été ainsi possible de différencier l'œdème de la réaction immédiate dans le derme des modifications plus tardives entraînées par la perte d'eau au niveau des couches cornées.

L'étude du relief cutané humain après une exposition à des rayonnements $\gamma$ a été abordée d'une manière très simplifiée par Morris et al. [19], pour estimer la densité des glandes eccrines fonctionnelles résiduelles après radiothérapie, sur une cohorte de patients dont près de la moitié présentait des télangiectasies associées à une atrophie cutanée définitive.

Nous rapportons ici des résultats préliminaires concernant les modifications radioinduites de certains paramètres décrivant le relief cutané, dans deux modèles expérimentaux d'accident de surexposition, c'est-à-dire $24 \mathrm{~h}$ après différentes doses d'irradiations aiguës localisées au strontium-yttrium 90 (rayonnement $\beta$ ) et à l'iridium 192 (rayonnement $\gamma$ ).

\section{Matériel et méthodes}

\subsection{Animaux}

L'expérimentation réalisée ici chez le porc bénéficie de l'autorisation $n^{\circ} 3255$ de l'Office de protection animale du ministère de l'agriculture et de la forêt. Quatre porcs, Large White femelles pesant $50 \mathrm{~kg}$ environ, ont été utilisés pour cette expérimentation. Les porcs, logés en cages individuelles, sont soumis 
à $12 \mathrm{~h}$ de lumière artificielle et $12 \mathrm{~h}$ d'obscurité et reçoivent deux repas d'aliments concentrés par jour. Pour toute intervention, les animaux sont manipulés sous anesthésie afin de limiter le stress: prémédication par injection IM d'Imalgène 1000 (kétamine : $4 \mathrm{ml})$ puis anesthésie gazeuse $\left(66 \% \mathrm{O}_{2}+33 \%\right.$ $\mathrm{NO}_{2}$ avec $2 \%$ d'halothane) avec une surveillance permanente de la saturation artérielle en oxygène. Les animaux sont replacés dans leurs cages encore anesthésiés.

\subsection{Irradiation $\beta$}

Une irradiation localisée $\beta$ au strontium-yttrium 90 (porcs $n^{\circ}$ P39914, P39082 et P39083) a été appliquée sur la peau préalablement rasée du flanc droit de chaque animal, au moyen d'un irradiateur original construit au SPR du CE-Saclay et renfermant une source scellée série II [3, 9] Amersham type SICQ $2575\left(\mathrm{n}^{\circ}\right.$ de série : $\left.8031 \mathrm{BA}\right)$ de $4,2 \mathrm{~cm}$ de diamètre de ${ }^{90} \mathrm{Sr}-90 \mathrm{Y}$ $\left(E_{\max } \cdot \beta: 2,28 \mathrm{MeV}\right)$. L'activité était de $1,85 \mathrm{GBq}$ au 16.07 .87 et les valeurs d'étalonnage du débit de dose, avec et sans écran diffuseur au 01.07 .88 , étaient respectivement de 52,1 et de $33,6 \mathrm{cGy} / \mathrm{h}$, à $30 \mathrm{~cm}$ sous $7 \mathrm{mg} / \mathrm{cm}^{2}$ (certificat d'étalonnage LMRI $\mathrm{n}^{\circ} 4856,1988$ ). La protection en plexiglass de $1 \mathrm{~cm}$ d'épaisseur rajoutée autour du porte-source permet d'appliquer celui-ci sans pression sur les flancs de l'animal en libérant une surface collimatée de $4 \mathrm{~cm}$ de diamètre sur la peau, sans risque d'irradiation latérale. La distance sourcepeau $\left(d_{\text {sp }}\right)$ est alors de $2,6 \mathrm{~cm}$. Les mesures de dosimétrie (LMRI, CE-Saclay), réalisées dans la même géométrie avec une chambre à extrapolation, ont permis de définir un débit de dose de $50,95 \mathrm{~Gy} / \mathrm{h}\left(d_{\mathrm{sp}}: 2,6 \mathrm{~cm}\right)$ sous $7 \mathrm{mg} / \mathrm{cm}^{2}$, et nous avons utilisé les doses uniques de 32, 64 et $96 \mathrm{~Gy}$ à la basale de l'épiderme, sur une surface de $4 \mathrm{~cm}$ de diamètre. Dans la peau exposée à la source, l'épiderme, le derme, l'hypoderme et le tissu adipeux sous-cutané ont été exposés au rayonnement compte tenu de l'énergie et de la pénétration du rayonnement $\beta$ du ${ }^{90} \mathrm{Sr}-90 \mathrm{Y}[20,21]$.

\subsection{Irradiation $\gamma$}

Une irradiation localisée $\gamma$ à l'iridium 192 (porc $\mathrm{n}^{\circ}$ P39983) a été appliquée sur la peau préalablement rasée de la face externe de la cuisse droite, au milieu et en arrière du fémur. Au niveau du point d'application de la source, l'épiderme, le derme, l'hypoderme, le tissu adipeux sous-cutané, le fascia lata, l'aponévrose et le muscle biceps femoris ont été exposés au rayonnement. Les conditions expérimentales d'irradiation et les mesures de dosimétrie ont déjà été publiées en détail $[6,17]$. Brièvement, l'irradiation par la source collimatée de ${ }^{192} \operatorname{Ir}\left(E_{\text {moy }} \gamma: 0,38 \mathrm{MeV}\right)$ est caractérisée par la dose reçue à la peau, choisie comme rendement $100 \%$. Le rendement en profondeur à 1,2 et $3 \mathrm{~cm}$ de profondeur correspond respectivement à 46,25 et $15 \%$ de la dose peau. Le débit de dose était $8 \mathrm{~Gy} / \mathrm{min}$ pour une $d_{\mathrm{sp}}$ de $1,7 \mathrm{~cm}$ et nous avons utilisé la dose unique de $160 \mathrm{~Gy} /$ peau sur une surface irradiée de $2 \mathrm{~cm}$ de diamètre. 


\subsection{Empreintes - analyse}

Un jour après irradiation, une empreinte de $3 \mathrm{~mm}$ d'épaisseur de pâte à empreintes aux silicones (Silflo, Flexico developments Ltd) est réalisée sur chaque zone irradiée, préalablement nettoyée et délimitée avec un crayon feutre à encre indélébile qui marquera l'empreinte, et débordant largement sur la peau saine adjacente. Après polymérisation ( 5 min environ), l'empreinte de résine Silflo est décollée de la peau, numérotée et archivée, et une réplique "en positif" de l'empreinte est réalisée en résine araldite afin de permettre l'analyse bi- et tridimensionnelle par lecture du relief par profilométrie mécanique à contact ou par une diode laser $(\lambda=0,788 \mu \mathrm{m})$ [24-25]. La sensibilité est alors de $\pm 0,5 \mu \mathrm{m}$ en profilométrie à contact ou de $\pm 3 \mu \mathrm{m}$ en profilométrie par diode laser, pour un déplacement vertical de $1000 \mu \mathrm{m}$.

Sur chaque empreinte, 4 zones de $5 \times 5 \mathrm{~mm}$ (pas: $20 \mu \mathrm{m}$; matrice de $250 \times 250$ points) (P39983) ou 4 zones de $10 \times 10 \mathrm{~mm}$ (pas : $20 \mu \mathrm{m}$; matrice de $500 \times 500$ points) (P39082, P39914 et P39083) sont analysées pour les mesures tridimensionnelles de peau irradiée et de peau normale adjacente (Fig. 1), et un profil de longueur totale de 80 à $130 \mathrm{~mm}$ est extrait de chacune des surfaces précédentes (Fig. 2 et 3 ). Ainsi, deux séries de paramètres de quantification sont disponibles: des paramètres de surface issus de l'analyse $3 \mathrm{D}$ et des paramètres de profilométrie $2 \mathrm{D}$. Ces paramètres $(\mathrm{Tab}$. I) sont calculés sur la totalité de la surface d'analyse, après application d'un filtrage passe-haut (filtre gaussien de $1 \times 1 \mathrm{~mm}$ ) afin d'éliminer les éventuels défauts de forme ou d'ondulations.

Pour chaque surface de peau étudiée, irradiée ou non, un calcul de significativité sur la différence des valeurs moyennes des paramètres décrivant les zones irradiées et témoin a été réalisé, basé sur le calcul du coefficient $t$, pour un intervalle de confiance de $95 \%$.

\section{Résultats - Discussion}

\subsection{Observation macroscopique}

Un jour après irradiation et sur animaux vigiles, la zone irradiée à 32 Gy $\beta$ ne présente aucune réaction clinique. Cette lésion ne dépassera pas le stade de l'érythème discret 8 semaines après irradiation.

Les zones irradiées à 64 et 96 Gy $\beta$ présentent un érythème distinct sans réaction cedémateuse cliniquement décelable dans le derme superficiel. Pour ces deux doses d'irradiation, les lésions évolueront vers l'épithéliite exsudative puis la nécrose cutanée ( $6^{\mathrm{e}}$ à $8^{\mathrm{e}}$ semaines) après une période asymptomatique d'environ 4 semaines.

La zone irradiée à 160 Gy $\gamma$ présente un érythème prononcé, associé à un derme légèrement œdématié. La lésion évoluera rapidement vers l'épithéliite exsudative et l'ulcération ( 5 semaines). 


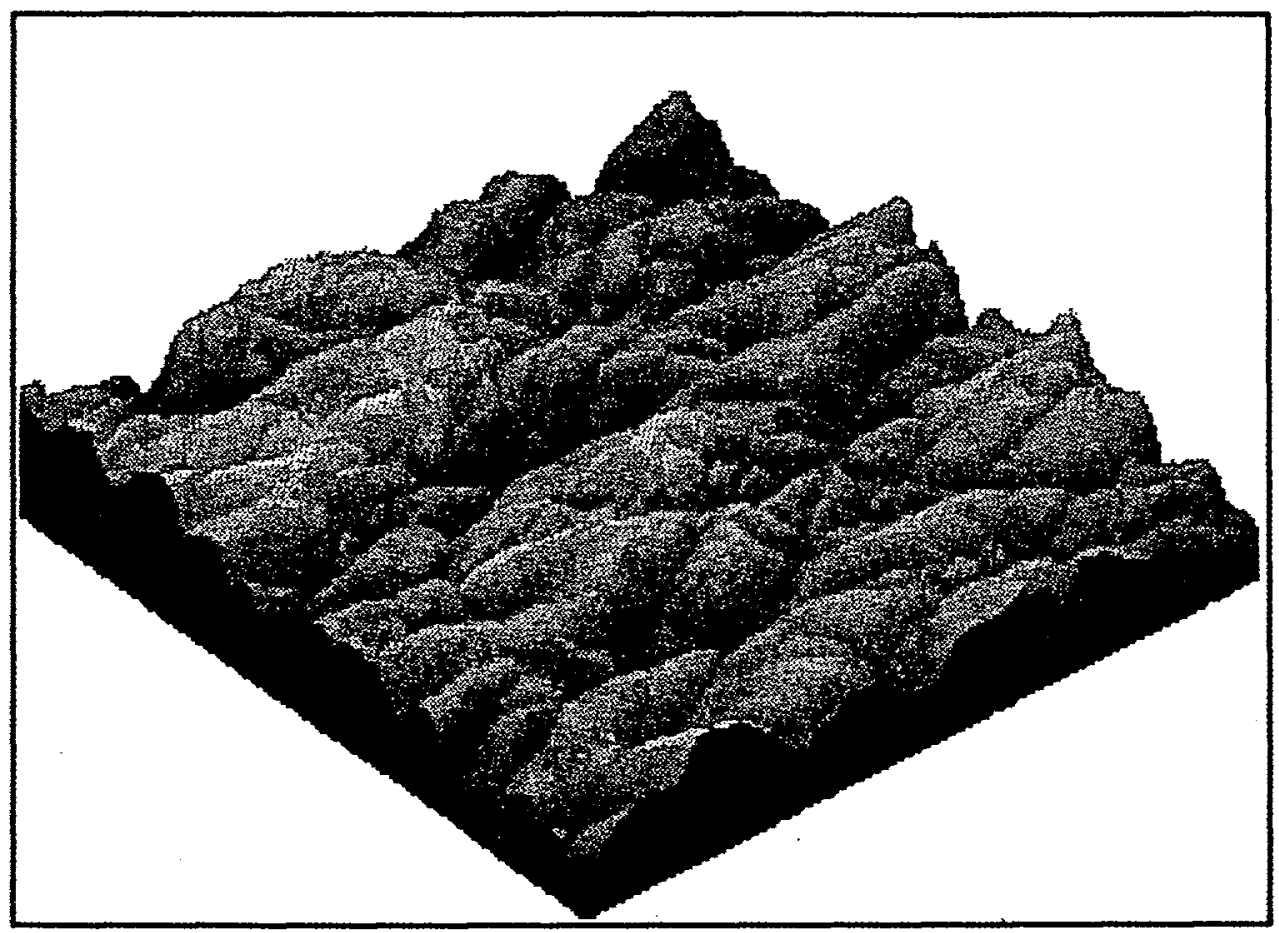

Fig. 1 - Exemple de surface d'analyse (animal 39083). Aire disponible après filtrage des défauts de forme : 9,3 $\times 9,3 \mathrm{~mm}^{2}$; dénivelée maximale: $600 \mu \mathrm{m} ; 217200$ points de mesure.

An example of surface analysis (animal 39083). Area available after filtration of shape defaults : $9.3 \times 9.3 \mathrm{~mm}^{2}$, maximum height difference : $600 \mu \mathrm{m} ; 217200$ measure points.

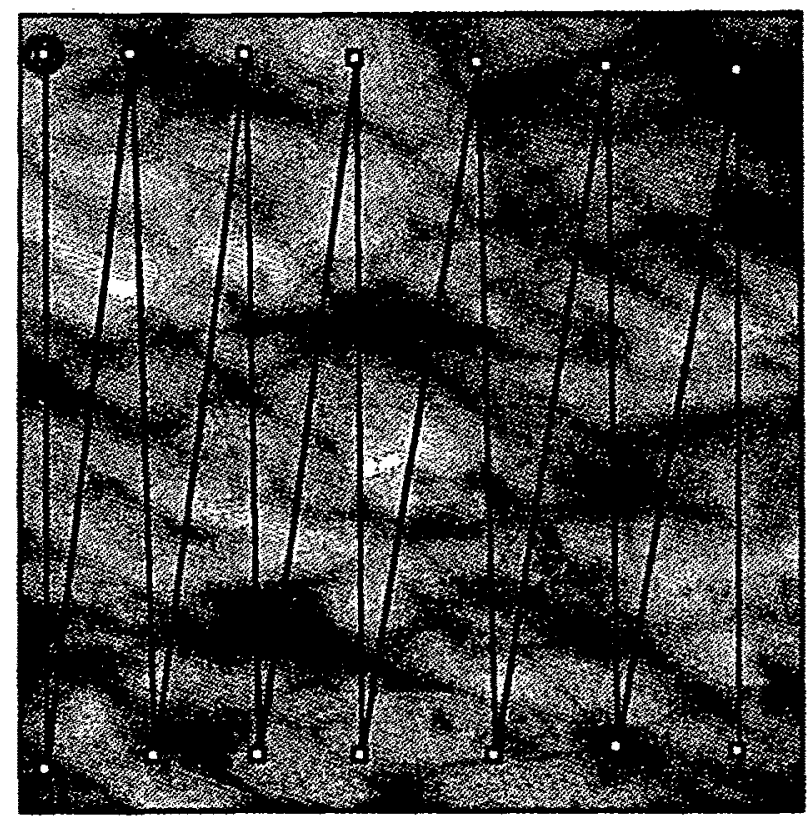

Fig. 2 - Vue de dessus de la surface d'analyse et extraction d'un profil selon une ligne brisée. Longueur du profil : $110 \mathrm{~mm}$; pas d'échantillonnage : $20 \mu \mathrm{m}$.

Top view of the surface analysed and extraction of a profile according to a broken line. Profile length : $110 \mathrm{~mm}$; sampling step : $20 \mu \mathrm{m}$. 


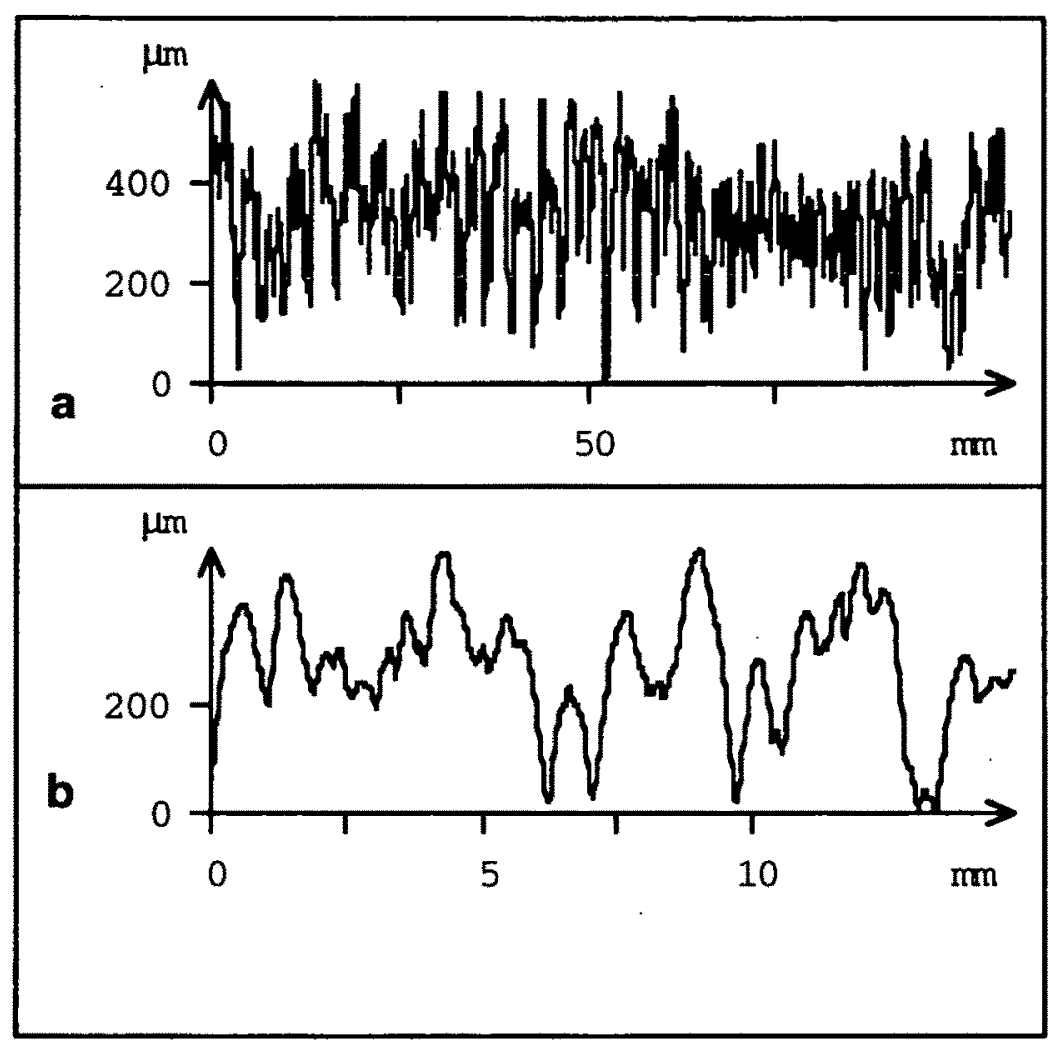

Fig. 3 - a) Profil extrait de la surface ; b) détail du profil.

a) Surface profile; b) detail of the profile.

Après une quinzaine de minutes d'anesthésie, temps nécessaire à la préparation des animaux et des zones où les empreintes seront relevées, l'érythème observé dans la zone irradiée à $64 \mathrm{~Gy} \beta$ a disparu, alors que les notations cliniques observées dans les zones irradiées à $96 \mathrm{~Gy} \beta$ et $160 \mathrm{~Gy} \gamma$ sont inchangées.

L'application et le décollement après polymérisation de la pâte à empreinte ne modifient pas la coloration de la peau en zone saine ni l'intensité de l'érythème en zones irradiées.

\subsection{Analyse du relief cutané}

\section{a) Zones témoins}

Parmi les 4 empreintes relevées, les différences maximales sont observées entre le P39983 $(S R a=24,8 \mu \mathrm{m})$ et le P39083 $(S R a=50,9 \mu \mathrm{m})$ (Tab. II). La moyenne élevée des valeurs absolues des hauteurs par rapport au plan moyen de la surface de la peau normale $(S R a)$ est corrélée à une grande différence pic-creux rencontrée $(S R t)$, ce qui entraîne une augmentation de l'écart-type de la distribution des hauteurs $(S R q)$. Cette corrélation directe se retrouve dans 
Tableau I

Paramètres décrivant l'étude du relief cutané.

Ces paramètres sont définis dans [12]

Parameters describing the study of the skin relief, as described in [12]

\begin{tabular}{|c|c|}
\hline $4 x$ & W Analyse de surface \\
\hline$S R a$ & $\begin{array}{l}\text { moyenne des valeurs absolues des hauteurs de tous les points de la surface, } \\
\text { hauteur évaluée relativement au plan moyen }\end{array}$ \\
\hline$S R t$ & valeur de la plus grande différence pic - creux rencontrée \\
\hline$S R q$ & écart-type de la distribution des hauteurs \\
\hline & Anase de profiometrie \\
\hline$R a$ & $\begin{array}{l}\text { moyenne des valeurs absolues des hauteurs des points du profil extraites } \\
\text { de la surface selon une ligne brisée }\end{array}$ \\
\hline Rtm & $\begin{array}{l}\text { moyenne des plus grandes différences pic-creux rencontrées sur chacun } \\
\text { des segments du profil de longueur } 8 \mathrm{~mm} \text { (largeur du filtre ou largeur } \\
\text { de base) }\end{array}$ \\
\hline Rpm & $\begin{array}{l}\text { moyenne des hauteurs maximales rencontrées, au-dessus de la ligne } \\
\text { moyenne, sur chacun des segments du profil }\end{array}$ \\
\hline$R q$ & écart-type de la distribution des hauteurs \\
\hline$S m$ & $\begin{array}{l}\text { moyenne des écartements horizontaux entre les saillies du profil, } \\
\text { écartements mesurés au niveau de la ligne moyenne }\end{array}$ \\
\hline$S$ & $\begin{array}{l}\text { moyenne des écartements horizontaux entre les saillies du profil, } \\
\text { quelle que soit leur position verticale }\end{array}$ \\
\hline
\end{tabular}

les mêmes paramètres décrivant la profilométrie. Cette hétérogénéité apparente des paramètres décrivant l'amplitude verticale du relief de peau normale de sujets de même sexe, âge et lignées parentales peut être liée à des caractéristiques anatomiques entre la face externe de la cuisse (P39983) et le flanc où ces valeurs semblent plus homogènes chez les 3 autres animaux.

A l'inverse, les paramètres décrivant l'amplitude horizontale des plis ( $\mathrm{Sm}$ et $S$ ) semblent plus homogène d'un sujet à l'autre.

\section{b) Zones irradiées}

Dans les trois zones irradiées au rayonnement $\beta$ et quelle que soit la dose, la moyenne des valeurs absolues des hauteurs de tous les pics du relief par rap- 
Tableau II

Modifications radioinduites des paramètres de relief cutané, 24 h après irradiation

Radioinduced modifications of the skin relief parameters, $24 \mathrm{~h}$ after irradiation

\begin{tabular}{|c|c|c|c|c|c|c|c|c|c|c|}
\hline Pore no & $\begin{array}{l}\text { Dose (Gy) } \\
\text { et rayoninement }\end{array}$ & Spa & $\begin{array}{l}S R t \\
(\mu m) \\
(\mu m)\end{array}$ & $\begin{array}{r}\text { SRq } \\
(\mu \mathrm{m}) \\
\end{array}$ & $\begin{array}{l}R a \\
(\mu \mathrm{m})\end{array}$ & $R\left(r_{m}\right)$ & $\begin{array}{r}R p m \\
(p m)^{2}\end{array}$ & $\begin{array}{l}R q \\
(\mu m){ }^{2}\end{array}$ & $\begin{array}{l}S_{m} \\
(\min ) \\
(\operatorname{mon})\end{array}$ & $\begin{array}{r}S \\
(\mu \mathrm{m})\end{array}$ \\
\hline & 0 & 39,2 & 377 & 48,6 & 52,9 & 293 & 123 & 65,8 & 1044 & 499 \\
\hline P39082 & 32 Gy $\beta$ & 33,3 & 370 & 41,6 & 50,2 & 272 & 121 & 62,4 & 1203 & 515 \\
\hline & & $*$ & ns & $*$ & $*$ & $*$ & ns & $*$ & $*$ & ns \\
\hline & Différence (\%) & $-15,1$ & $-1,9$ & $-14,4$ & $-5,1$ & $-7,2$ & $-1,6$ & $-5,2$ & $+15,2$ & $+\mathbf{3 , 2}$ \\
\hline & & & & & & & & & & \\
\hline & $\mathbf{0}$ & 30,5 & 313 & 37,8 & 63,6 & 299 & 146 & 75,5 & 934 & 564 \\
\hline P39914 & $64 \mathrm{~Gy} \beta$ & 25,3 & 193 & 31,1 & 54,1 & 265 & 121 & 65,9 & 900 & 536 \\
\hline & & $*$ & $*$ & $*$ & $*$ & $*$ & ns & $*$ & ns & ns \\
\hline & Différence (\%) & $-17,0$ & $-38,1$ & $-\mathbf{1 7 , 7}$ & $-14,9$ & $-11,4$ & $-17,5$ & $-\mathbf{1 2 , 7}$ & $-3,6$ & $-5,0$ \\
\hline & 0 & 50,9 & 568 & 63,8 & 80,8 & 450 & 195 & 102 & 1288 & 585 \\
\hline P39083 & $96 \mathrm{~Gy} \beta$ & 43 & 391 & 53,1 & 67,2 & 364 & 153 & 83 & 1229 & 519 \\
\hline & & $*$ & $*$ & $*$ & $*$ & $*$ & $*$ & $*$ & ns & ns \\
\hline & Différence (\%) & $-15,5$ & $-31,2$ & $-16,8$ & $-16,8$ & $-19,1$ & $-21,5$ & $-18,5$ & $-4,6$ & $-11,3$ \\
\hline & & & & & & & & 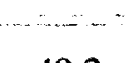 & (n) & \\
\hline & 0 & 24,8 & 266 & 31,8 & 38,7 & 236 & 93,2 & 49,2 & 1301 & 674 \\
\hline P39983 & $160 \mathrm{~Gy} \gamma$ & 22,9 & 245 & 29,3 & 37,4 & 210 & 85,7 & 47,1 & 1409 & 683 \\
\hline & m.m. & $*$ & $*$ & $*$ & ns & $*$ & $*$ & * & ns & ns \\
\hline & Différence (\%) & $-7,7$ & $-7,8$ & $-7,9$ & $-3,4$ & $-10,8$ & $-8,0$ & $-4,3$ & $+8,3$ & $+1,4$ \\
\hline
\end{tabular}

* : significatif à $p<0,05 ;$ ns : non significatif.

port au plan moyen $(S R a)$ est significativement abaissée d'environ $15 \%$. Cette observation semble indépendante de l'absence $(32 \mathrm{~Gy})$ ou de la présence (64 et $96 \mathrm{~Gy})$ de réaction érythémateuse radioinduite.

Les diminutions les plus importantes des paramètres de quantification verticale (SRt, Ra, Rtm et $R p m$ ) varient de $10 \%$ à $40 \%$ excepté pour la zone irradiée 32 Gy $\beta$ qui n'est pas significativement différente de son propre témoin. A l'inverse, cette seconde observation peut être liée à l'aspect clinique de la peau. En effet, l'érythème cutané (64 et $96 \mathrm{~Gy}$ ), reflet de la vasodilatation capillaire du derme papillaire anatomiquement liée à la couche basale de l'épiderme, peut induire quelques augmentations ponctuelles de l'amplitude verticale du relief de l'épiderme, qui persistent même si cet érythème a été atténué ou a disparu au cours de l'anesthésie qui précède le relevé d'empreinte. Cependant, il est à noter que l'effet vasodilatateur d'une anesthésie gazeuse est général et peut s'exercer avec des intensités variables, aussi bien en tissu sain qu'en tissu irradié. 
Dans la zone irradiée à $160 \mathrm{~Gy} \gamma$, la diminution d'amplitude verticale d'environ $8 \%$, bien que significativement différente de la zone témoin, est moins importante que dans les autres zones. Outre la raison d'ordre anatomique, probablement en partie responsable de cette différence, la réaction œéémateuse associée à l'érythème peut, en partie, être à l'origine d'une dilatation des structures de l'épiderme et du derme papillaire suffisante pour atténuer les variations radioinduites du microrelief cutané.

Les paramètres décrivant l'amplitude horizontale des plis ( $S m$ et $S$ ) augmentés chez le $\mathrm{P} 39082$ et le $\mathrm{P} 39983$ ne permettent pas de conclure à une variation significative.

En résumé, nous avons utilisé une technique rapide et atraumatique d'exploration du microrelief du tissu cutané, dans deux modèles expérimentaux d'irradiation aiguë accidentelle par rayonnements $\beta$ et $\gamma$. Cette étude préliminaire a mis en évidence une diminution des paramètres d'amplitude verticale de même grandeur relative, dès $24 \mathrm{~h}$ après une irradiation à une dose aussi faible que 32 Gy $\beta$, qui ne présentera aucune évolution radiopathologique cliniquement décelable. Ce résultat original mérite d'être complété par une étude sur une série chronologique portant sur plusieurs individus, afin de tenter de dégager une relation entre les variations radioinduites des paramètres d'amplitudes verticale et horizontale des plis du relief cutané, prenant en compte les nombreux paramètres absents de cette étude préliminaire, et la dose d'irradiation. Une relation dose-effet permettrait alors de valider cette méthode d'analyse d'empreinte comme moyen de diagnostic ou de dosimétrie biologique de la peau irradiée en cas de surexposition accidentelle.

\section{Remerciements}

Nous tenons à remercier chaleureusement MM. C. Chazot et D. Delacroix du SPRI, CE-Saclay, pour leur accueil et la mise à disposition de leur irradiateur équipé de sa source de ${ }^{90}{ }^{9}-90 Y$, et M. J-J. Leplat pour sa compétence technique lors des anesthésies des animaux. Nous tenons également à adresser un remerciement particulier à Mme C. Costa et $M$. B. Chauvenet du LMRI-CE-Saclay pour la gentillesse avec laquelle ils ont pu surmonter les difficultés occasionnées par les mesures dosimétriques du ${ }^{90} \mathrm{Sr}-90 \mathrm{Y}$.

Ce travail bénéficie d'une aide du Comité de radioprotection d'EDF (contrat 1J3802/D348).

\section{RÉFÉRENCES}

[1] BESSIS S., GROLLIER J.F., PASERO P. - Modifications du relief cutané provoquées par le rayonnement ultraviolet. Int. J. Cosmet. Sci., 1984, 6, 1-11.

[2] CHALANSONNET A., EL KAMOUNI N., BRIGUET A., DABURON F., LEFAIX J-L. Mise en évidence des effets d'une irradiation aiguë localisée par imagerie de résonance magnétique du tissu cutané. Radioprotection, 1993, 28(4), 411-421. 
[3] CHARTIER J-L., CUTARELLA D., ITIÉ C. - Characterization of the radiation fields of beta secondary standards with extended area sources. Radiat. Prot. Dosim., 1991, 39(1/3), 115-118.

[4] COOK T.H. - Profilometry of skin : useful tool for the substantiation of cosmetic efficacy. J. Soc. Cosmet. Chem., 1980, 31, 339-359.

[5] COOK T.H., CRAFT T.J., BRUNELL R.L. - Quantification of the skin topography by skin profilometry. Int. J. Cosmet. Sci., 1982, 4, 195-205.

[6] DABURON F., LEFAIX J-L., REMY J., FAYART G., TRICAUD Y. - Intérêt et limites des mesures thermographiques microondes pour le diagnostic et le pronostic des irradiations aiguës localisées chez le porc. Radioprotection, 1985, 20(3), 207-225.

[7] DABURON F., LEFAIX J-L., LAVAL M., MARTIN J-L., LE BAS J-F., WAKSMANN B. - Intérêt de la tomographie $X$ et $R M N$ pour le diagnostic précoce des irradiations localisées. Etude expérimentale chez le porc. J. Biophys. Bioméc., 1986, 10(2), 89-92.

[8] DABURON F., LEFAIX J-L., HOFFSCHIR D., FAYART G. - Dosimétrie biologique des irradiations aiguës localisées. Radioprotection, 1991, 26, suppl. 1, 265-282.

[9] FRANCIS T.M., BÖHM J., CHARTIER J-L., CHRISTENSEN P. - Experience gained on extrapolation chamber measurement techniques from an intercomparison exercise conducted with a ${ }^{147}$ Pm source. Radiat. Prot. Dosim., 1991, 39(1/3), 109-114.

[10] HOPEWELL J.W. - The skin : its structure and response to ionizing radiation. Int. J. Radiat. Biol., 1990, 57, 751-773.

[11] INTERNATIONAL COMMISSION ON RADIOLOGICAL PROTECTION (ICRP) - Biological basis for dose limitation in the skin. (ICRP Publication 59). Annals of the ICRP, 1991, 22 (2) $1-104$.

[12] INTERNATIONAL STANDARDIZATION ORGANIZATION (ISO) - Rugosité de surface. Terminologie ; partie 1 : surface et ses paramètres; partie 2 : mesurage de la rugosité de surface. Norme ISO 4287-1 et 2, 1984.

[13] LEFAIX J-L., VEROLA O., DABURON F., BROCHERIOU C. - Les lésions cutanées et musculaires après irradiation aiguë chez le porc. Ann. Pathol., 1985, 5(4/5), 249-258.

[14] LEFAIX J-L., VEROLA O., DABURON F., BROCHÉRIOU C. - Early changes in irradiated skeletal muscle : a histo-enzymological and immunocytochemical study. Brit. J. Radiol. 1986, suppl. 19, 109-113.

[15] LEFAIX J-L., DABURON F., TRICAUD Y. - Evolution radiopathologique spontanée et après traitement médical dans deux modèles d'accident d'irradiation localisée. Bull. Cancer/Radiothér., 1992, 79, 189-198.

[16] LEFAIX J-L., DABURON F., FAYART G. - Dosimetry study of an accidental overexposure to ${ }^{192}$ Ir gamma rays. Health Phys., 1992, 63 (6) 692-694.

[17] LEFAIX J-L., MARTIN M., TRICAUD Y., DABURON F. - Muscular fibrosis induced after pig skin irradiation with single doses of ${ }^{192}$ Ir $\gamma$-rays. Brit. J. Radiol, 1993, 66, 537-544.

[18] MAKKI S., BARBENEL J.C., AGACHE P. - A quantitative method for the assessment of microtopography of human skin. Acta Derm. Venereol. (Stockholm) 1979, 59, 281-291.

[19] MORRIS W.J., DISCHE S., MOTT G. - A pilot study of a method of estimating the number of functional eccrine sweat glands in irradiated human skin. Radiother. Oncol., 1992, 25, 49-55.

[20] PEEL D.M., HOPEWELL J.W., WELLS J., CHARLES M.W. - Nonstochastic effects of different energy beta emitters on pig skin. Radiat. Res., 1984, 99, 372-382.

[21] PEEL D.M., HOPEWELL J.W., WELLS J., CHARLES M.W. - Late nonstochastic changes on pig skin after beta irradiation. Radiat. Res., 1985, 101, 491-496.

[22] VIDAL H. - Les rayonnements ionisants. Applications médicales et industrielles. Radioprotection, 1994, 29(2), 213-229.

[23] XIE Y., MIGNOT J. - Measurement of skin relief by two- and three-dimensional profilometry. In : "Aging skin, properties and functional changes" (J-L. Lévêque, P.A. Agache, Eds). New York : Marcel Dekker, 1993, 133-178. 
[24] XIE Y., ASSOUL M., MIGNOT J. - Morphological and spectral analysis of human skin surface topography. Innov. Technol. Biol. Med., 1993, 14(5), 575-587.

[25] ZAHIDI M., ASSOUL M., MIGNOT J., BELLATON B. - A fast 2D/3D optical profilometer for wide range topographical measurement. Wear, 1993, 165, 197-203.

[26] ZAHOUANI H., ASSOUL M., JANOD P., MIGNOT J. - Theoretical and experimental study of wound healing: application to leg ulcers. Med. Biol. Eng. Comput., 1992, 30, 234-239. 